\title{
Continuous spectra in high-harmonic generation driven by multicycle laser pulses
}

\author{
W. Holgado, ${ }^{1, *}$ C. Hernández-García, ${ }^{1,2}$ B. Alonso, ${ }^{1,3}$ M. Miranda,,${ }^{3,4}$ F. Silva, ${ }^{3}$ L. Plaja, ${ }^{1}$ H. Crespo, ${ }^{3}$ and I. J. Sola ${ }^{1}$ \\ ${ }^{1}$ Grupo de Investigación en Aplicaciones del Láser y Fotónica, Universidad de Salamanca, E-37008 Salamanca, Spain \\ ${ }^{2}$ JILA, University of Colorado Boulder, Boulder, Colorado 80309-0440, USA \\ ${ }^{3}$ IFIMUP-IN and Departamento de Física e Astronomia, Faculdade de Ciências, Universidade do Porto, \\ Rua do Campo Alegre 687, 4169-007 Porto, Portugal \\ ${ }^{4}$ Department of Physics, Lund University, P.O. Box 118, SE-221 00 Lund, Sweden
}

(Received 9 October 2015; published 13 January 2016)

\begin{abstract}
We present observations of the emission of XUV continua in the 20-37-eV region by high-harmonic generation with 4-7-fs pulses focused onto a Kr gas jet. The underlying mechanism relies on coherent control of the relative delays and phases between individually generated attosecond pulses, achievable by adjusting the chirp of the driving pulses and the interaction geometry. Under adequate negative chirp and phase-matching conditions, the resulting interpulse interference yields a continuum XUV spectrum, which is due to both microscopic and macroscopic (propagation) contributions. This technique opens the route for modifying the phase of individual attosecond pulses and for the coherent synthesis of XUV continua from multicycle driving laser pulses without the need of an isolated attosecond burst.
\end{abstract}

DOI: 10.1103/PhysRevA.93.013816

\section{INTRODUCTION}

High-order-harmonic generation (HHG) is a process where, from the nonlinear interaction of an intense laser pulse with an atom or a molecule, new frequencies in the extreme ultraviolet (XUV) and soft-X-ray range are generated. This can be described by a semiclassical three-step model [1,2] and the radiation is emitted as a train of bursts with durations down to the attosecond scale, commonly presenting an odd-harmonic comblike spectrum.

Since the advent of HHG and subsequently of attosecond science, the generation of continua in the XUV range has been of great interest. This was originally associated with the process of emission of a single attosecond light pulse [3,4], but it also presents interest as a broadband source for spectroscopic applications. For instance, characterization of the optical properties of materials and components in the XUV range is challenging, usually requiring synchrotron facilities for broadband spectral measurements. Recently, typical comblike HHG spectra have also proved to be a tool for XUV optical characterization. For instance, Goh et al. have applied a HHG source for diffraction grating characterization [5]. An HHG source has also been applied to mirror reflectance analysis, complementing synchrotron source assisted measurements of reflectivity with spectral phase characterization by employing the odd-harmonic comblike spectrum from HHG and an attosecond pulse reconstruction technique [6]. In this case, the comblike spectrum provides access to the phase information at some wavelengths (the odd harmonics) but, in this context, a continuous coherent XUV spectrum may represent an interesting source capable of accessing a broader and more complete spectral region. Such spectra are foreseen also as a key for extending spectral interferometric techniques, such as optical coherence tomography, to the XUV domain [7]. Unlike comblike spectra, continuous coherent XUV spectra enable easier observation of spectral interference over a continuous range and the coherence of $\mathrm{HHG}$ radiation allows for the

\footnotetext{
*warein@usal.es
}

measurement of not only amplitude changes but also of phase variations during a certain experiment or material interaction. Therefore, a coherent continuum XUV source enables a more complete characterization (amplitude and phase) in this spectral range. As a last example, the powerful techniques of time-resolved and transient absorption XUV spectroscopy are behind very active research aimed at understanding the electron dynamics in atoms and molecules (see, e.g., [8-10]) although the use of a probe beam with a comblike spectra from HHG limits the analyzed region to that covered by the harmonics. Goulielmakis et al. have shown that by using an isolated attosecond pulse, the technique can give more information, mainly due to the fact that it corresponds to a continuum in the spectral domain [11].

To date, most of the strategies to obtain XUV continua have relied on isolating an attosecond pulse at the microscopic level (i.e., a single atom). This implies the occurrence of a single ionization-flight-recombination event, which is only possible if the driving field pulse is as short as the period of the central frequency or, in the case of few-cycle pulses, if HHG is confined by gating techniques. Different strategies have been proposed, based on carrier-envelope-phase-stabilized few-cycle driving fields, namely, spectral selection of the cutoff [3], polarization gating [4,12], and double optical gating [13], among others. The drawback of such techniques is the decrease of the generation efficiency [14], making it difficult to apply them to low-energy driving fields, such as the new high-repetition-rate $\mu \mathrm{J}$-level optical parametric chirpedpulse amplification sources [15,16]. Macroscopic phenomena can also be relevant to isolate attosecond pulses [17]. For instance, different techniques that make use of phase-matching conditions have been proposed, such as the use of flat-top beams at the focus [18], ionization gating [19,20], or tilted pulse beams [21]. Recently, phase matching on high-pressure gases has been proposed to obtain a single XUV burst [22]. However, all the mentioned techniques generate XUV continua through the emission of an isolated attosecond pulse.

In previous works the effect of the chirp of the driving pulse over HHG generated on gas targets, which is directly related 


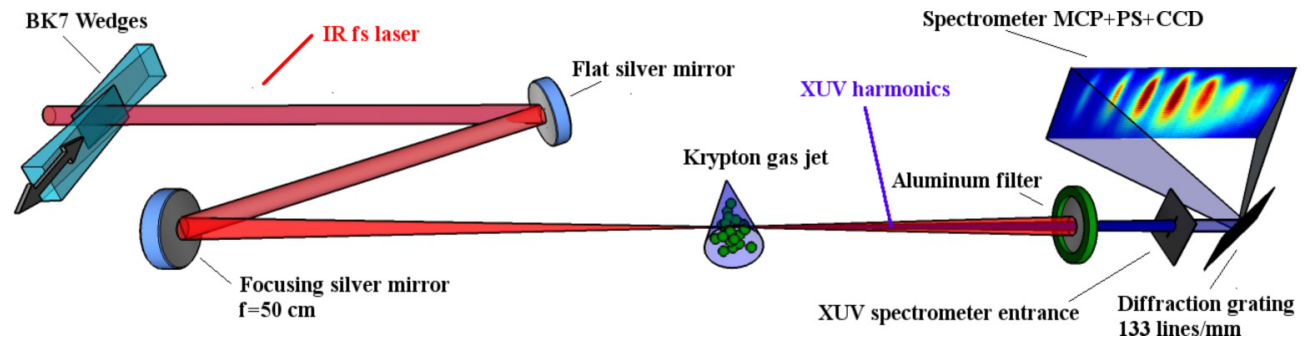

FIG. 1. Diagram of the experimental setup. A pair of BK7 wedges, with an angle of $8^{\circ}$, is placed in the beam path. One of them is placed on a translation stage, allowing us to modify the amount of BK7 in the path and hence the dispersion of the output pulses. The IR pulses are then focused with a spherical silver mirror into a krypton gas jet, where XUV high-order harmonics are generated. The infrared beam, together with the low-order harmonics (up to the 11th), is filtered by an aluminum foil filter (150 nm thick) while the higher-order harmonics are diffracted into an MCP, phosphor screen (PS) and CCD combination.

to the delay between each attosecond burst, has been studied $[23,24]$. The continuous spectrum emerged in the cutoff region for IR pulses that were negatively or highly positively chirped, while the plateau remained insensible to changes in the IR chirp. On the other hand, Lee et al. measured continuous spectra in HHG performed in neon [25] for harmonic orders between 60 and $90(61-136 \mathrm{eV})$ and driving pulses that were negatively and positively chirped.

In this work we explore the generation of XUV continua driven by multicycle laser pulses, by altering the relative delay and phase of a train of attosecond bursts, without going through the complexities of generating an isolated pulse. We present experimental results, corroborated by theoretical simulations, that demonstrate the emission of XUV continua ranging from 20 to $37 \mathrm{eV}$ as a result of the combination of microscopic and macroscopic physics. In contrast to previous works, the generated XUV continua lie in the low HHG plateau (wavelengths between 30 and $60 \mathrm{~nm}$ ). Furthermore, we present theoretical results that prove the influence of propagation in the resulting spectra.

In the first part of this paper we present the experimental setup and results. In the second part, theoretical simulations are presented, discussed, and compared to experiments, whereby the nature of the obtained XUV continua is inferred.

\section{EXPERIMENTAL RESULTS}

In the experiments we use a $1-\mathrm{kHz}$ Ti:sapphire chirpedpulse amplification amplifier (FemtoPower Compact PRO $\mathrm{CEP}$, Femtolasers $\mathrm{GmbH}$ ) delivering pulses with a Fouriertransform-limited duration of 25 fs [full width at half maximum (FWHM)] and energy up to $0.9 \mathrm{~mJ}$. The output pulses are spectrally broadened in a hollow-core fiber (HCF) with an inner diameter of $250 \mu \mathrm{m}$ and 1-m length. The HCF is filled with argon at 1 bar pressure. The carrier-envelope phase (CEP) is stabilized in the oscillator, with a CEP rms of $100 \mathrm{mrad}$ in the fast loop, and well preserved during the amplification and post-compression processes.

The resulting broadband pulses are then sent through a chirped mirror and glass wedge compressor that forms part of a dispersion-scan [26] pulse measurement and compression system. By compensating for the spectral phase with ten bounces off double-angle chirped mirrors (Ultrafast Innovations $\mathrm{GmbH}$; nominal group delay dispersion of $-40 \mathrm{fs}^{2}$ per pair at $800 \mathrm{~nm}$ and minimum reflectance of 99\%) and the addition of normal dispersive material (a pair of BK7 wedges, with a group velocity dispersion of $44.6 \mathrm{fs}^{2} / \mathrm{mm}$ ), few-cycle pulses are obtained. Using this setup, we can routinely achieve pulse durations down to below 4 fs $[27,28]$. Depending on the gas pressure, the pulse duration can be tuned in a certain range. In our experiments we work with pulse durations from 4 to 7 fs (FWHM), as determined with dispersion-scan measurements.

The laser pulses are then focused by a spherical silver mirror $(f=500 \mathrm{~mm}$ ) into a pulsed $\mathrm{Kr}$ gas jet, as shown in Fig. 1, with an estimated intensity on target of $2 \times 10^{14} \mathrm{~W} / \mathrm{cm}^{2}$. Since krypton has lower $I_{p}$ than argon, we use $\mathrm{Kr}$ to obtain a larger HHG signal [29]. The spherical mirror is placed on a translation stage, so the position of the focus can be controlled. The pulse enters the vacuum chamber through a $0.5-\mathrm{mm}$-thick fused-silica window, situated close to the focusing mirror to avoid possible nonlinear effects. High-harmonic generation is driven in a $\mathrm{Kr}$ gas jet ( 5 bars of backing pressure) using a nozzle of 500- $\mu \mathrm{m}$ diameter. The pressure reached inside the vacuum chamber where the high-order harmonics are generated is around $5 \times 10^{-3}$ mbar. A 150 -nm-thick aluminum foil is used to filter the IR radiation and the lower harmonics. The HHG spectra are characterized with a grazing-incidence Rowland circle XUV spectrometer (Model 248/310G, McPherson Inc.), of $1-\mathrm{m}$ radius and 133 -grooves/mm spherical diffraction grating.

The gas jet is placed $4 \mathrm{~mm}$ and $2 \mathrm{~mm}$ before the focus position for 4-fs and 7-fs pulses, respectively. The gas jet is placed before the focus to select favorable transversal phasematching conditions for the observation of CEP-dependent HHG [30]. A dispersion scan is performed on the driving pulses by finely translating one of the BK7 wedges. When the insertion of glass (BK7) in the beam path is increased, the pulse becomes positively chirped. Therefore, HHG is studied as a function of the spectral phase of the incident beam. Furthermore, if the scan is fine enough, this chirp scan becomes a CEP scan, since the CEP of the infrared pulse is modified by $\pi$ when propagating through $25.2 \mu \mathrm{m}$ of BK7.

In Fig. 2(a) we present the variation of the HHG spectra as a function of the BK7 insertion. The map reveals how crucial the effect of the IR spectral phase on the HHG is. As depicted, the narrower structures appear when the generating beam is positively chirped, while at negative chirping the spectra 

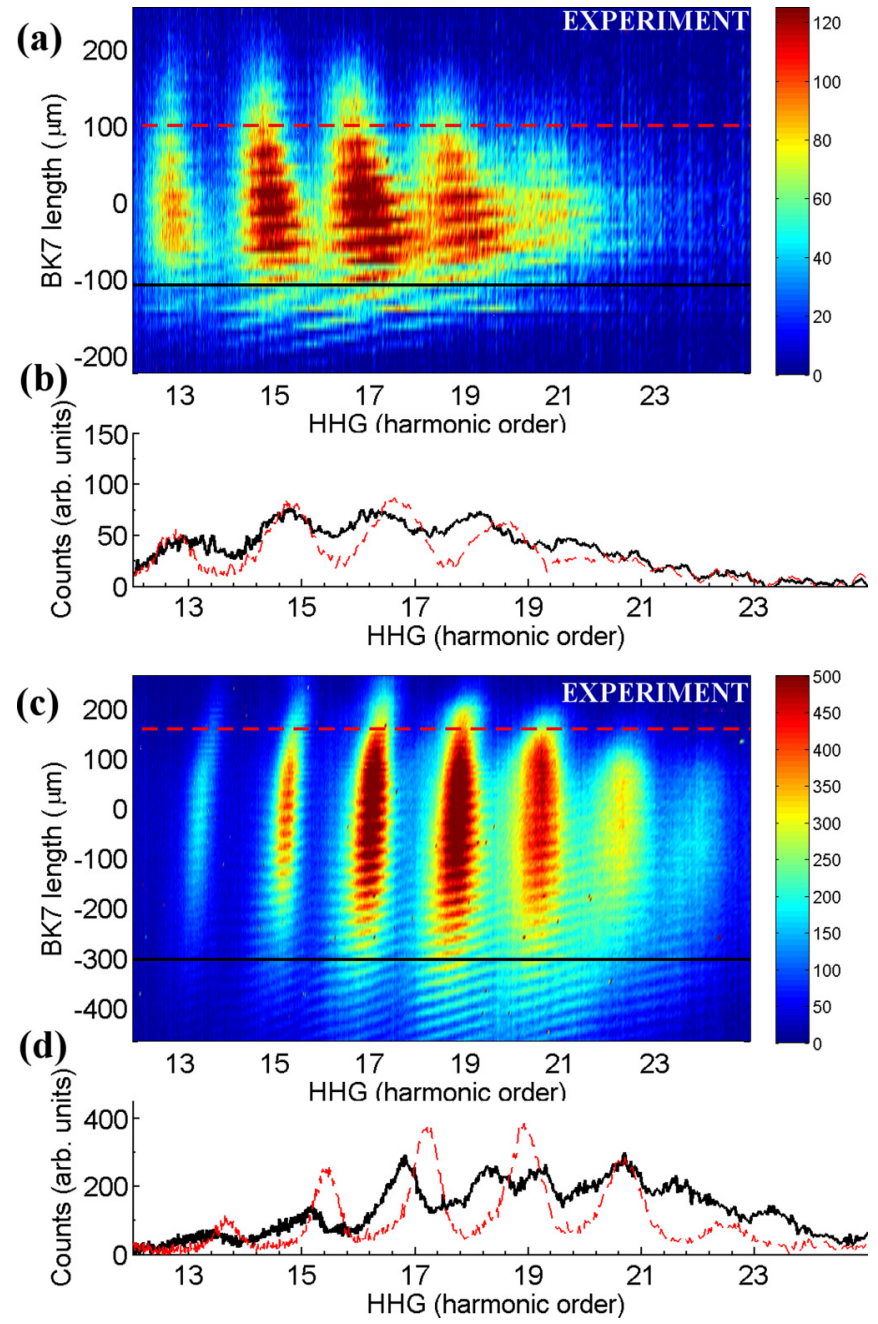

FIG. 2. Measured HHG dependence on BK7 insertion for (a) 4-fs pulses and (b) 7-fs pulses. When the insertion is finely varied, a CEP dependence can be observed in the HHG spectra in the form of fringes. The $y$ axes in (a) and (c) give the amount of BK7 insertion. Below each scan, in (b) and (d), XUV spectra are shown for the BK7 insertion that results in negative chirp (black solid line, shown as a black cut in the corresponding chirp-scan trace) and in positive chirp (red dashed lines).

broaden, until eventually becoming continua covering over 12 harmonic orders (around $17 \mathrm{eV}$ ). The observed fringes denote the CEP dependence of the HHG (i.e., for a short range of BK7 insertion, the dispersion scan becomes a CEP scan).

In Fig. 2(c) we show the CEP scan for a 7-fs driving pulse. The range of dispersion values generating an efficient HHG signal is narrower for 4-fs pulses [Fig. 2(a)] than for 7-fs pulses, since the spectral content of the former is broader than that of the latter, but in both cases the continuum appears for negative chirp [black solid lines in Figs. 2(b) and 2(d)].

In Fig. 2(b) we show the continuum achieved with 4-fs laser pulses for a BK7 insertion of $-108 \mu \mathrm{m}$ (black solid line), i.e., when $108 \mu \mathrm{m}$ are removed from the Fourier-limit condition. In order to quantify the quality or smoothness of the achieved continuum, we calculate the corresponding contrast for each harmonic order. Contrast is defined as

$$
C=\frac{I_{\max }-I_{\min }}{I_{\max }+I_{\min }} .
$$

We compute this value for regions of two orders of harmonics. The mean contrast in the case of $4 \mathrm{fs}$, in a range from the 13th to the 21st harmonic, is 0.43 . In the case of 7 -fs laser pulses, for a BK7 insertion of $-303 \mu \mathrm{m}$ [shown in Fig. 2(d), black solid line] and a spectrum between harmonic orders 15 th and 23 th, the mean contrast is 0.40 . Even though these are not totally smooth (unmodulated) continua, there are no wavelengths with zero signal in this range. Contrarily, in the case of positive chirp [red dashed line in Figs. 2(b) and 2(d)], these values are 0.62 for $4 \mathrm{fs}$ (insertion of $100 \mu \mathrm{m}$ ) and 0.76 for $7 \mathrm{fs}$ (insertion of $152 \mu \mathrm{m}$ ).

It is already known that HHG shows a spectral broadening (narrowing) when negatively (positively) chirped pulses are employed [23-25,31], contrarily to what occurs in the self-phase-modulation process [31]. However, the continua described in those works were only achieved for very-highharmonic orders (in $\mathrm{Ne},>70 \mathrm{eV}$ ). Calegari et al. [32] reported continua in Xe using 5 -fs pulses focused into a gas cell in a high-intensity regime $\left(2.5 \times 10^{15} \mathrm{~W} / \mathrm{cm}^{2}\right)$ when selecting the so-called short quantum paths. According to the authors, this effect is introduced by the plasma-induced chirp of the driving field as one main factor, yielding a single attosecond pulse in the time domain. In our case, we perform a scan over chirp to fully understand its effect.

\section{THEORETICAL SIMULATIONS}

In the observed case, continua generated for negatively chirped driving pulse conditions appear dramatically in a relatively-low-energy region (20-37 eV), covering both the HHG plateau and the cutoff. Similar behavior was observed in [16].

The appearance of continua when using negatively chirped driving pulses is reproduced by our calculations. We compute harmonic propagation using a method based on the electromagnetic field propagator [33]. The dipole acceleration of each elementary source is computed using an extension of the strong-field approximation method [34]. Due to the lack of contrasted Coulomb potentials for krypton, we perform our HHG calculations in argon (which has similar ionization potential), using the Coulomb potential given in [35]. Propagation effects of the fundamental field, including plasma and neutral dispersion as well as time-dependent group velocity walkoff, are all taken into account. The absorption of the harmonics in the gas is modeled using Beer's law. The agreement between the model and theory when phase matching is a relevant factor was already tested in Refs. [22,30,36,37].

Interestingly, the origin of the continuum behavior shown in Fig. 2 can be found at both the microscopic and macroscopic levels. In Fig. 3(a) we represent the simulated microscopic single-atom spectra driven in argon versus BK7 insertion, where the driving laser pulse is modeled by a $\cos ^{2}$ envelope function of 4.3-fs FWHM, with $2.5 \times 10^{14} \mathrm{~W} / \mathrm{cm}^{2}$ peak intensity and a central wavelength of $720 \mathrm{~nm}$. By changing the BK7 insertion, a standard harmonic spectrum structure is visible for positive and slightly negative chirp cases. However, 

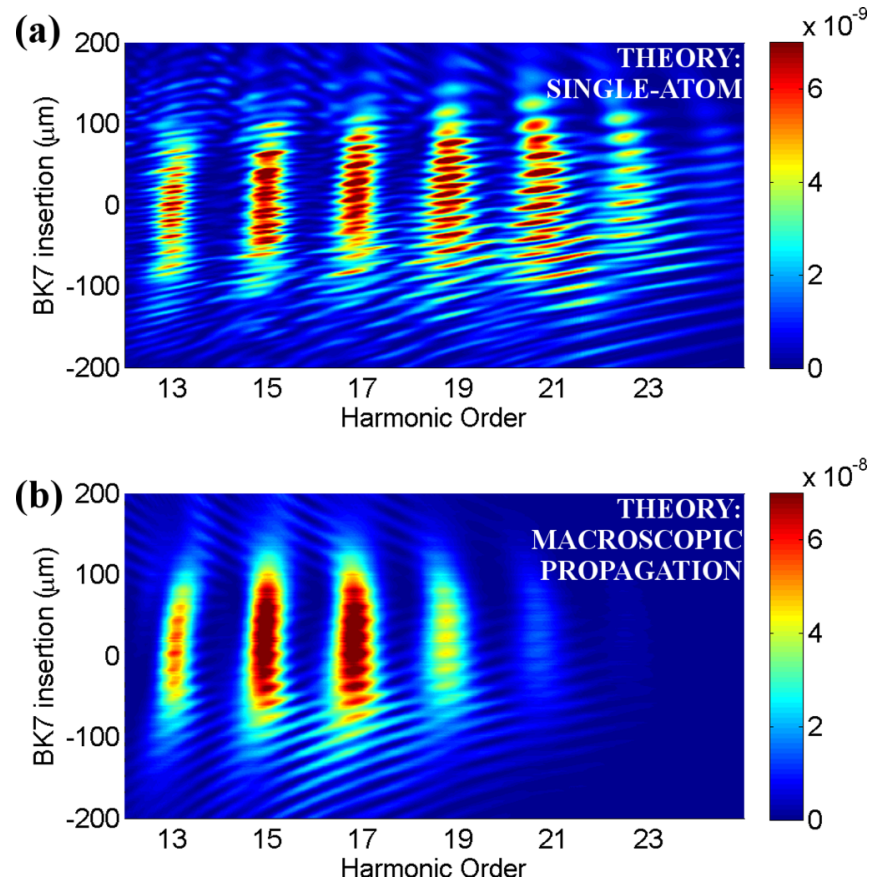

FIG. 3. Simulated HHG (a) from the single-atom response and (b) including macroscopic propagation effects for different chirps of the driving IR pulse. Chirp is scanned by the insertion of BK7, in a similar way as in the experiment. Zero insertion means a Fourier-limit generating pulse ( $4.3 \mathrm{fs}$ in this case).

for sufficiently negatively chirped input pulses, the HHG spectral structure changes and spectral contributions appear out of the odd-harmonic positions. Although the behavior of the single-atom CEP scan is similar to that observed experimentally, it does not fully reproduce our results.

Propagation effects alter further these spectral structures, as shown in Fig. 3(b), obtained for similar experimental focusing conditions. We use a Gaussian beam, whose waist at the entrance plane of the 40 -cm-focal-length lens is $2.5 \mathrm{~mm}$, focused into a gas jet placed $2 \mathrm{~mm}$ before the focus position. The gas jet is modeled by a Gaussian distribution along the gas propagation direction with 1-mm FWHM and peak density of 10 mbar. We show the harmonics as detected on axis. Cutoff harmonics are lost during propagation [38] and also odd harmonics show less chirp-dependent modulation than the single-atom response in the positive-chirp region. In the case of negative chirp, variations in the odd-harmonic structure are still observed, but appear smoother than in the single-atom case. The modulated spectrum obtained in the single-atom response is filled when propagation is taken into account.

\section{DISCUSSION}

For further insight into the nature of the continua, we have analyzed the numerical simulations. For the sake of clarity, we compare a negatively chirped IR pulse ( $-100 \mu \mathrm{m}$, Fig. 4$)$, a Fourier-limit pulse (Fig. 5), and a positively chirped pulse (94 $\mu \mathrm{m}$, Fig. 6). In the harmonic emission spectrum [Figs. 4(a), 5(a), and 6(a)], blue lines denote the single-atom response, while red lines represent calculations including propagation. In agreement with experiments, the negative chirp case yields

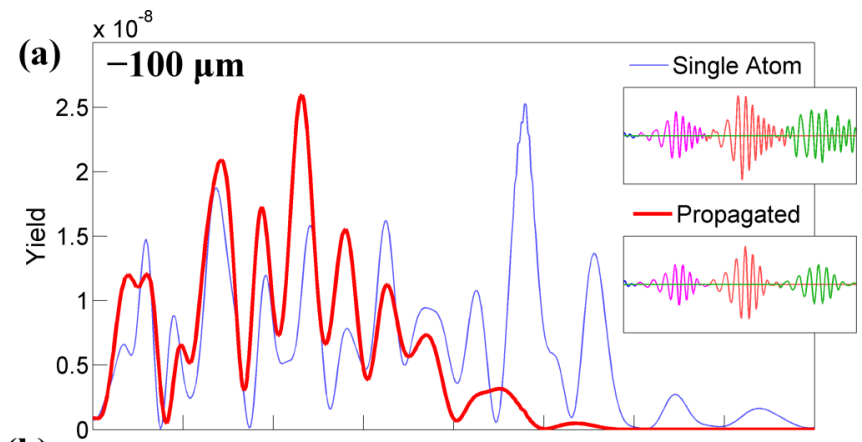

(b)

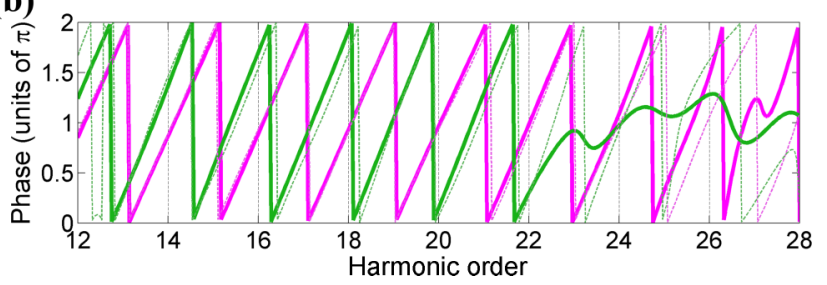

FIG. 4. (a) Simulated XUV spectra when $100 \mu \mathrm{m}$ of BK7 have been removed from the Fourier-limit condition (4.3-fs pulse duration at the Fourier limit). The thin blue line denotes the single-atom response while the thick red line shows the collective spectrum. The corresponding time evolution is shown in an inset for both cases, single-atom simulations and propagated calculations, denoting at different colors the main individual attosecond pulses. (b) Corresponding spectral phase differences between the first and second attosecond pulses (thick violet line for propagated and thin dashed violet for single-atom pulses) and between the second and third pulses (thick green line for propagated and thin dashed green for single atom) are shown.

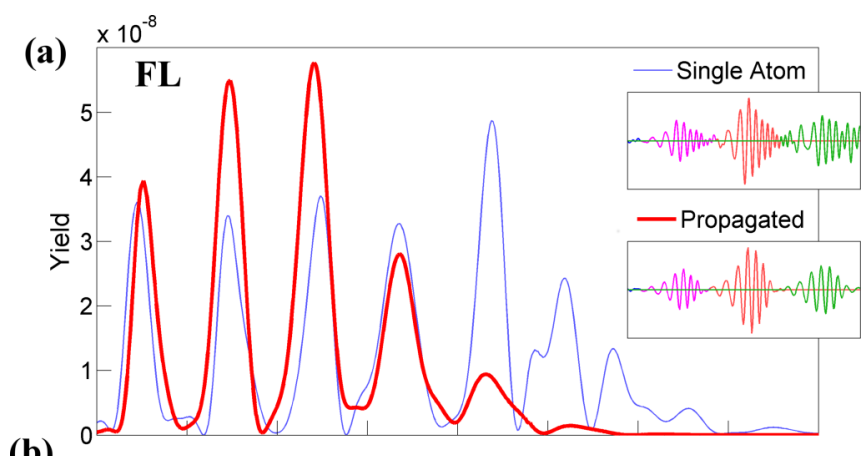

(b)

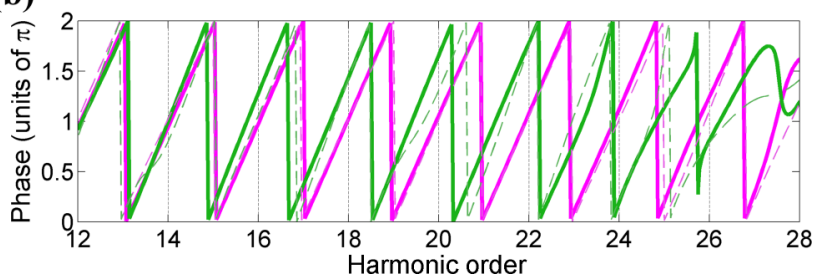

FIG. 5. (a) Simulated XUV spectra for Fourier-limited (FL) driving pulses with a duration of $4 \mathrm{fs}$. The thin blue line is for the single-atom response and the thick red line shows the result for when the propagation is considered. The insets show the corresponding time profiles for single-atom and propagated simulations. (b) Spectral phase differences between the first and second attosecond pulses (thick violet line for propagated and dashed line for single-atom pulses) and between the second and third pulses (thick and dashed green lines). 


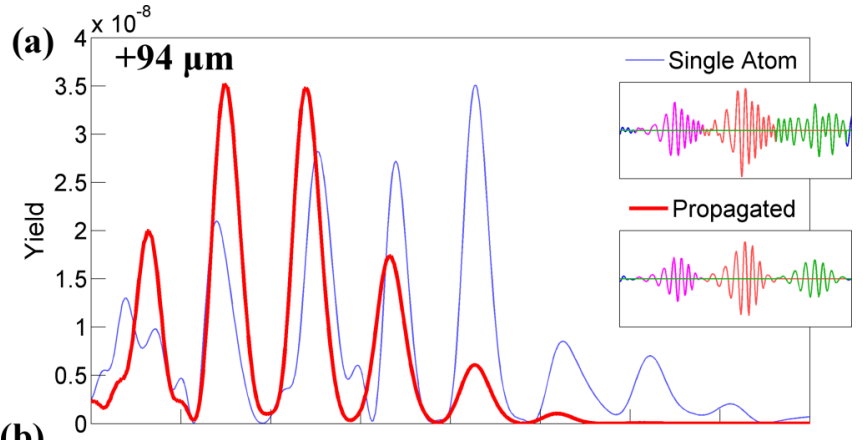

(b)

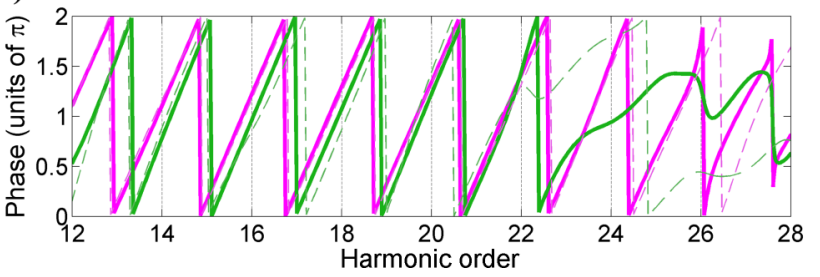

FIG. 6. (a) Simulated XUV spectra for positively chirped driving pulses (94 $\mu \mathrm{m}$ of BK7 added from the Fourier-limit condition). The thin blue line is for single-atom pulses and the thick red line is for the collective spectrum. The insets show the corresponding time profiles for single-atom and propagated simulations. (b) Spectral phase differences between the first and second attosecond pulses (violet lines) and between the second and third pulses (green lines).

a continuous spectrum [Fig. 4(a)] and spectra driven by unchirped or positively chirped IR fields show the usual HHG comblike structure composed of odd harmonics [Figs. 5(a) and 6(a)]. The insets show the temporal structure for both the single-atom and the propagated cases. The different colors denote the main attosecond pulses within the train, amounting to 3 in this case.

The role of negative chirp in inducing changes over the harmonic structure at the single-atom response level has been analyzed in HHG from solid targets [39], observing a nonconstant time separation between the attosecond pulses. This may lead to a Moiré pattern in the spectral domain, merging some harmonics and changing their wavelength but in general maintaining a peaked structure. In Ref. [39], negative chirp in the IR driving field was identified as a major factor, since it enhances time-delay emission differences, as demonstrated in Ref. [40] (in this case considering HHG from gas targets). In our case, this phenomenon is observed (see the insets in Figs. 4-6), but notably the continuum is also associated with a particular phase difference among the individual attosecond pulses [Figs. 4(b), 5(b), and 6(b), where the violet (green) line represents the phase difference between the first and second (second and third) attosecond pulses]. The XUV spectra corresponding to the different chirp cases have been analyzed and described as the result of spectral interference of the single bursts, as suggested in Refs. [16,41].

For a negatively chirped driving field [Fig. 4(b)] the spectral phase difference between consecutive attosecond pulses within the pulse train [Fig. 4(a) inset] yields a continuum. Note that the continuum structure appears when the phase difference between the first (pink) and second (red) pulses differs from that between the second (red) and third (green) pulses. In other words, at wavelengths where dephasing of a pair of pulses is $\pi+2 m \pi$ rad ( $m$ being natural), a minimum is found in the spectrum due to destructive interference and maxima arise from constructive interference when the dephasing is $2 m \pi \mathrm{rad}$. When the dephasings among the pulse pairs are similar [as it happens when using a Fourier limit or a positively chirped driving field, Figs. 5(b) and 6(b)], a sharp odd-harmonic structure arises. Contrarily, when dephasings do not agree and even have a difference of $\pi \mathrm{rad}$ [Fig. 4(b)], a more continuous structure emerges from interference [Fig. 4(a)]. Note that the dephasing is established at the single-atom level [thin lines in Figs. 4(b), 5(b), and 6(b)] and is conserved when propagated (thick lines). The macroscopic addition and propagation of different XUV fields reinforces the obtention of the continuous structure.

\section{CONCLUSION}

To conclude, spectral continua have been observed in the medium-energy range of high-harmonic spectra, for adequate focusing and IR pulse conditions. Our simulations reveal that the origin of the continua can be understood not as the emission of an isolated attosecond pulse, but as the interference of several pulses in a train with different dephasing between consecutive pairs of pulses. The particular phase difference yielding the continuum structure can be obtained through adjustment of the focus position, IR pulse chirp, and CEP, allowing a fine control of the resulting spectral interference pattern. Effects due to microscopic and macroscopic contributions were described and compared. From a practical point of view, this method enables obtaining continua in the XUV range without the need of pulse gating techniques, high intensities, or single-cycle driving IR pulses.

\section{ACKNOWLEDGMENTS}

We acknowledge support from a Marie Curie International Outgoing Fellowship within the EU Seventh Framework Programme for Research and Technological Development, under REA Grant Agreement No. 328334, support from Junta de Castilla y León (Project No. SA116U13, UIC016), Spanish MINECO (Grants No. FIS2009-09522, No. FIS201344174-P and No. FIS2015-71933-REDT), and Centro de Láseres Pulsados is gratefully acknowledged. This work was partly supported by Grant No. PTDC/FIS/122511/2010. F.S. acknowledges support from Grant No. SFRH/BD/69913/2010 and B.A. acknowledges support from Post-Doctoral fellowship No. SFRH/BPD/88424/2012. H.C. acknowledges support from Sabbatical Leave Grant SFRH/BSAB/105974/2015, from Fundação para a Ciência e Tecnologia, Portugal, cofunded by COMPETE and FEDER.
[1] P. B. Corkum, Phys. Rev. Lett. 71, 1994 (1993).

[2] K. J. Schafer, B. Yang, L. F. DiMauro, and K. C. Kulander, Phys. Rev. Lett. 70, 1599 (1993).
[3] M. Hentschel, R. Kienberger, C. Spielmann, G. A. Reider, N. Milosevic, T. Brabec, P. Corkum, U. Heinzmann, M. Drescher, and F. Krausz, Nature (London) 414, 509 (2001). 
[4] G. Sansone, E. Benedetti, F. Calegari, C. Vozzi, L. Avaldi, R. Flammini, L. Poletto, P. Villoresi, C. Altucci, R. Velotta, S. Stagira, S. De Silvestri, and M. Nisoli, Science 314, 443 (2006).

[5] S. J. Goh, H. J. M. Bastiaens, B. Vratzov, Q. Huang, F. Bijkerk, and K. J. Boller, Opt. Express 23, 4421 (2015).

[6] A. Morlens, R. López-Martens, O. Boyko, P. Zeitoun, P. Balcou, K. Varjú, E. Gustafsson, T. Remetter, A. L'Huillier, S. Kazamias et al., Opt. Express 31, 1558 (2006).

[7] S. Fuchs, A. Blinne, C. Rodel, U. Zastrau, V. Hilbert, M. Wunsche, J. Bierbach, E. Frumker, E. Forster, and G. Paulus, Appl. Phys. B 106, 789 (2012).

[8] M. Holler, F. Schapper, L. Gallmann, and U. Keller, Phys. Rev. Lett. 106, 123601 (2011).

[9] M. Chini, B. Zhao, H. Wang, Y. Cheng, S. X. Hu, and Z. Chang, Phys. Rev. Lett. 109, 073601 (2012).

[10] C. Ott, A. Kaldun, L. Argenti, P. Raith, K. Meyer, M. Laux, Y. Zhang, A. Blättermann, S. Hagstotz, T. Ding et al., Nature (London) 516, 374 (2014).

[11] E. Goulielmakis, Z. Loh, A. Wirth, R. Santra, N. Rohringer, V. S. Yakovlev, S. Zherebtsov, T. Pfeifer, A. M. Azzeer, M. F. Kling et al., Nature (London) 466, 739 (2010).

[12] I. J. Sola, E. Mevel, L. Elouga, E. Constant, V. Strelkov, L. Poletto, P. Villoresi, E. Benedetti, J. P. Caumes, S. Stagira et al., Nat. Phys. 2, 319 (2006).

[13] Z. Chang, Phys. Rev. A 76, 051403(R) (2007).

[14] G. Sansone, L. Poletto, and M. Nisoli, Nat. Photon. 5, 655 (2011).

[15] M. Krebs, S. Haedrich, S. Demmler, J. Rothhardt, A. Zaïr, L. Chipperfield, J. Limpert, and A. Tuennermann, Nat. Photon. 7, 555 (2013).

[16] P. Rudawski, A. Harth, C. Guo, E. Lorek, M. Miranda, C. M. Heyl, E. W. Larsen, J. Ahrens, O. Prochnow, T. Binhammer et al., Eur. Phys. J. D 69, 70 (2015).

[17] Y. Mairesse, A. de Bohan, L. Frasinski, H. Merdji, L. Dinu, P. Monchicourt, P. Breger, M. Kovačev, T. Auguste, B. Carré et al., Phys. Rev. Lett. 93, 163901 (2004).

[18] V. V. Strelkov, E. Mevel, and E. Constant, New J. Phys. 10, 083040 (2008).

[19] I. Thomann, A. Bahabad, X. Liu, R. Trebino, M. M. Murnane, and H. C. Kapteyn, Opt. Express 17, 4611 (2009).

[20] F. Ferrari, F. Calegari, M. Lucchini, C. Vozzi, S. Stagira, G. Sansone, and M. Nisoli, Nat. Photon. 4, 875 (2010).

[21] J. A. Wheeler, A. Borot, S. Monchocé, H. Vincenti, A. Ricci, A. Malvache, R. Lopez-Martens, and F. Quéré, Nat. Photon. 6, 829 (2012).

[22] M. Chen, C. Mancuso, C. Hernández-García, F. Dollar, B. Galloway, D. Popmintchev, P. Huang, B. Walker, L. Plaja, A.
Jarón-Becker et al., Proc. Natl. Acad. Sci. USA 111, E2361 (2014)

[23] Z. Chang, A. Rundquist, H. Wang, I. Christov, H. C. Kapteyn, and M. M. Murnane, Phys. Rev. A 58, R30(R) (1998).

[24] J. Zhou, J. Peatross, M. M. Murnane, H. C. Kapteyn, and I. P. Christov, Phys. Rev. Lett. 76, 752 (1996).

[25] D. G. Lee, J. H. Kim, K. H. Hong, and C. H. Nam, Phys. Rev. Lett. 87, 243902 (2001).

[26] M. Miranda, T. Fordell, C. Arnold, A. L'Huillier, and H. Crespo, Opt. Express 20, 688 (2012).

[27] B. Alonso, M. Miranda, F. Silva, V. Pervak, J. Rauschenberger, J. San Román, I. J. Sola, and H. Crespo, Appl. Phys. B 112, 105 (2013).

[28] F. Silva, M. Miranda, B. Alonso, J. Rauschenberger, V. Pervak, and H. Crespo, Opt. Express 22, 10181 (2014).

[29] Y. Liang, A. Talebpour, C. Y. Chien, S. Augst, and S. L. Chin, J. Phys. B 30, 1369 (1997).

[30] C. Hernández-García, W. Holgado, L. Plaja, B. Alonso, M. Miranda, F. Silva, L. Plaja, H. Crespo, and I. J. Sola, Opt. Express 23, 21497 (2015).

[31] P. Saliéres, A. L'Huillier, P. Antoine, and M. Lewenstein, Adv. At. Mol. Opt. Phys. 41, 83 (1999).

[32] F. Calegari, M. Lucchini, K. S. Kim, F. Ferrari, C. Vozzi, S. Stagira, G. Sansone, and M. Nisoli, Phys. Rev. A 84, 041802(R) (2011).

[33] C. Hernández-García, J. A. Pérez-Hernández, J. Ramos, E. C. Jarque, L. Roso, and L. Plaja, Phys. Rev. A 82, 033432 (2010).

[34] J. A. Pérez-Hernández, L. Roso, and L. Plaja, Laser Phys. 19, 1581 (2009).

[35] X. M. Tong and C. D. Lin, J. Phys. B 38, 2593 (2005).

[36] C. Hernández-García, I. J. Sola, and L. Plaja, Phys. Rev. A 88, 043848 (2013)

[37] M. Kretschmar, C. Hernández-García, D. S. Steingrube, L. Plaja, U. Morgner, and M. Kovačev, Phys. Rev. A 88, 013805 (2013).

[38] A. L'Huillier, M. Lewenstein, P. Salières, P. Balcou, M. Y. Ivanov, J. Larsson, and C. G. Wahlström, Phys. Rev. A 48, R3433(R) (1993).

[39] A. Borot, A. Malvache, X. Chen, A. Jullien, J. Geindre, P. Audebert, G. Mourou, F. Quéré, and R. Lopez-Martens, Nat. Phys. 8, 416 (2012).

[40] K. Varjú, Y. Mairesse, B. Carré, M. B. Gaarde, P. Johnsson, S. Kazamias, R. López-Martens, J. Mauritsson, K. J. Schafer, P. H. Balcou et al., J. Mod. Opt. 52, 379 (2005).

[41] C. Ott, M. Schönwald, P. Raith, A. Kaldun, G. Sansone, M. Krüger, P. Hommelhoff, Y. Patil, Y. Zhang, K. Meyer, M. Laux, and T. Pfeifer, New J. Phys. 15, 073031 (2013). 